\title{
28 Research Square \\ Red Clover Root-Associated Microbiota is Shaped by Geographic Location and Choice of Farming System
}

Shridhar Jambagi

Swedish University of Agricultural Sciences

Kristian Persson Hodén

Swedish University of Agricultural Sciences

Linda Öhlund

Lantmännen Lantbruk

Christina Dixelius ( $\sim$ Christina.Dixelius@slu.se )

Swedish University of Agricultural Sciences

\section{Research Article}

Keywords: Co-occurrence networks, Farming systems, Geographic locations, Rhizobium, Root-associated microbiota, Trifolium pratense

Posted Date: March 21st, 2022

DOI: https://doi.org/10.21203/rs.3.rs-1380141/v2

License: (c) (i) This work is licensed under a Creative Commons Attribution 4.0 International License. Read Full License 


\section{Abstract \\ Background}

Red clover (Trifolium pratense), a crop constrained by weak persistence, is the number one forage legume in Northern Europe. Whether plant loss is a sign of an increasing number of known or new pathogens, a decreasing occurrence of beneficial soil microorganisms or other factors is unclear. Here, we collected red clover root samples from Swedish conventional and organic farming sites located between latitudes $55^{\circ} \mathrm{N}$ and $66^{\circ} \mathrm{N}$ and analyzed their prokaryotic and eukaryotic communities using amplicon sequencing. We compared the organism diversity, differential abundance, hub taxa and impact of Rhizobium on fungal pathogens in relation to geographic location and farming practices.

\section{Results}

We found that microbial biodiversity was lowest in samples from southern Sweden and on conventional farms. Rhizobium was the most prevalent bacterial genus, followed by Sphingomonas, Mucilaginibacter, Flavobacterium and the unclassified Chloroflexi group KD4-96. The Leptodontidium, Cladosporium, Clonostachys and Tetracladium fungal genera known for endophytic, ectomycorrhizal, saprotrophic and mycoparasitic lifestyles were also frequently observed in all samples. Sixty-two potential pathogenic fungi were identified with a bias toward grass pathogens and a higher abundance in samples from conventional farms. To gain insights into the cooccurrence patterns of red clover rootassociated communities, intrakingdom and interkingdom networks were constructed. Bacterial networks were dominated by known beneficial groups of bacterial species. The interaction between Rhizobium leguminosarum bv. trifolii and Aeromicrobium sp. was strongly positive in the north but negative in the south. Different co-occurrence responses were observed when fungal and bacterial data were combined, as exemplified by the interactions with Clonostachys rosea, a beneficial fungus that fluctuated in our dataset and was positively associated with species in Enterobacterales and Aeromicrobium in the north but negatively with species in the south.

\section{Conclusions}

We show that the microbial community is mainly shaped by geographic location and management procedures. $R$. leguminosarum bv. trifolii is a keystone species in samples from the northern region and Swedish organic farms. This Rhizobium species is negatively associated with all fungal pathogenic species recognized in this study. A number of previously unknown fungal species are identified, particularly those known to produce mycotoxins, which are of major concern for crop productivity and feed safety.

\section{Background}

The sustainable use of resources for food and feed production is of increasing importance to meet demands from growing populations, shrinking agricultural land and requests for high-quality and nutritious products [1, 2]. A number of factors determine the productivity of each crop, and different management regimes are applied worldwide. Intense production systems are commonly associated with high resource and economical inputs but also with high losses in terms of nutrient leakage and the release of greenhouse gases [3]. Conversely, yields are higher from conventional farming [4]. The balance between the choice of agricultural management (technical-based), biological preconditions (soil type, water, temperature, light) and farming traditions, among other factors, varies greatly among countries and regions. 
The implementation of sustainable cropping systems highlights the importance of integrating legumes into crop rotation schemes [5]. Fabaceae is a species-rich plant family, but only a very small fraction of the approximately 19,000 species has been domesticated and used as a source for grains and forages. Concerns regarding climate change and the use of natural resources, such as in the production of mineral fertilizers, have revived interest in increasing the use of forage legume crops. Leys are associated with many advantages and ecosystem services, including nitrogen fixation, improved soil structure, and promotion of genetic diversity through insect pollination [6].

Red clover is a perennial species that is commonly grown intercropped with forage grasses to optimize the nutrient values of animal feed [7]. In Sweden, leys make up to $42 \%$ of the total acreage [8]; thus, red clover is by far the dominant legume crop. Soybean is not grown in northern Europe, and the import of soybean products is not regarded as a sustainable alternative to improve animal feed states. Current breeding goals of red clover comprise factors that influence yields, nutritional values, and persistence to withstand a range of stresses. Leys with red clover are aimed for two or three years of growth, hence the persistence of the red clover plants throughout the entire time period is important. The role of microbial soil biodiversity is here believed to be crucial but the knowledge of its potential status and impact is limited. Fungal diseases caused by Sclerotinia trifoliorum (clover rot) and Fusarium spp. (root rot) are observed in several locations [9] but not in all fields, highlighting the likelihood of new or hitherto unknown pathogenic microbes in soil.

In this work, we hypothesized that a higher microbial diversity is present in the southern part of the country, including an enrichment of potential pathogens and clear intra- and interkingdom competing patterns. The southern region of Sweden has a longer vegetation period than other parts of the country and hence an increased disease pressure. We further hypothesized that the choice of farm management would generate minor effects on the Rhizobium community. To address these questions regarding microbial community compositions and cooccurrence patterns in relation to geographic location and farming systems, red clover root samples were collected from south to north in selected places throughout Sweden.

\section{Materials And Methods}

\section{Field sample collection}

Eighty-nine field sites encompassing conventional and organic farms located between $55^{\circ} 50^{\prime} \mathrm{N}$ and $66^{\circ} 48^{\prime} \mathrm{N}$ were visited. In total, 260 samples were collected: 214 samples from 72 conventional and 46 from 17 organic farming sites (Additional file 1). From each field site, 2 to 4 samples were taken, separated by a maximal distance. Each sample consisted of 2 to 8 root systems of red clover with their surrounding soils. All samples were stored at $-20^{\circ} \mathrm{C}$ until DNA extraction.

\section{Sample preparation}

The samples were prepared as previously outlined [10] with a few modifications. The soil attached to the root system was carefully removed without damaging the roots. Root systems with closely adhering soil particles (rhizospheric soil) were used for microbiota profiling. From 2 to 4 randomly chosen root systems per sample, 5 to $6 \mathrm{~cm}$ root pieces (crown part) were cut into two halves and transferred into 50-ml Falcon tubes (Additional file 2: Fig. S1). The root pieces were thoroughly rinsed 3 times each with $30 \mathrm{ml}$ sterile ice-cold $\mathrm{H}_{2} \mathrm{O}$. The liquid fractions were collected and centrifuged for 15 min at $4500 \times g$ and $4^{\circ} \mathrm{C}$, and the resulting pellets were used for DNA extraction. The samples represent the rootassociated microbiota (RAM).

\section{Microbial community profiling}


DNA was extracted using a NucleoSpin Soil Kit (Macherey-Nagel, Germany) following the manufacturer's protocols. The quality and quantity of the sample DNA were checked on a Nanodrop 1000 spectrophotometer (Thermo Fisher Scientific). The 16S rRNA sequences were generated in two steps [11]. First, the PCR conditions and primers were applied as previously outlined $[12,13]$. Duplicated reactions were performed to reduce PCR biases. Amplified products were cleaned using Sera-Mag beads, pooled and used as a template for the second PCR step using the same conditions [12]. Duplicated amplicons were purified using an EZNA CyclePure Kit (Omega Bio-Tek, USA) and pooled into two libraries. The quality and quantity of the libraries were measured using a Nanodrop, Qubit 4 Fluorometer (Invitrogen) and Agilent 2100 Bioanalyzer. Sequencing was performed on an Illumina MiSeq with paired-end 250-bp reads using the MiSeq reagent kit v2 at SciLife Laboratory, National Genomics Infrastructure (NGI), SNP\&SEQ Technology Platform, Uppsala, Sweden. Demultiplexing of the reads was performed using Illumina software (version 2.6.2.1).

Eukaryotic ITS2 rDNA fragments were prepared by amplification using previously described conditions [14], including barcode information [15]. PCR was conducted in duplicate for each sample. Three purified amplicon pools were assembled by combining the barcoded DNA samples. The amplicon size distribution was prechecked using an Agilent 2100 Bioanalyzer. The libraries were prepared using the PacBio amplicon library protocol (Pacific Biosciences, Inc.) and sequenced on a Sequel instrument using three SMRT cells (SMRT Cell 1M v3) by SciLife Laboratory NGI (Uppsala, Sweden). PacBio circular consensus sequence reads were generated using SMRT Link v 8.0.0.79519 (Pacific Biosciences). For all sequencing primers, adapters and barcodes, see Additional file 2: Table S1 and Additional file 3.

\section{S rRNA gene and ITS2 rDNA read processing}

Paired 16S rRNA amplicon sequencing reads were processed by applying the DADA2 method [16] in QIIME2 version 2020.8 (https://qiime2.org/) by filtering and trimming to a fixed length of $250 \mathrm{bp}$, including quality checking and chimera removal. Next, filtered sequences were clustered into amplicon sequence variants (ASVs) at 100\% identity. Taxonomic assignment of ASVs was performed using SILVA database release 138 [17] in QIIME2 with the q2-feature-classifier plugin. The QIIME2-compatible preformatted SILVA reference sequence and taxonomy files were processed using RESCRIPt (http://doi.org/10.5281/zenodo.3891931). The output was used to train the taxonomic classifier by extracting the hypervariable V3-V4 region of the 16S rRNA gene. Multiple sequence alignment and phylogenetic reconstruction were carried out using MAFFT and FastTree $([18,19]$. Taxonomy-based filtering of ASV sequences was performed using the q2-taxa plugin to remove ASVs representing mitochondrial and chloroplast sequences. Samples were rarefied to an even depth of 11,184 reads to compensate for different sequencing depths.

ITS2 amplicon data were processed using various tools implemented in the PipeCraft v1.0 platform [20]. PacBio circular consensus sequence reads were quality filtered with Mothur 1.36.1 [21] (qwindowaverage = 30, qwindowsize = 50, minlength $=100$, maxambig $=0$, maxhomop $=12$ ). The resulting reads were demultiplexed based on the unique sequence identifiers using Mothur by allowing one mismatch to the barcode (bdiff $=1$ ) and two mismatches to the primer sequences (pdiff $=2$ ). Putative chimeric sequences were removed using vsearch ( 1 1.11.1;

github.com/torognes/vsearch) de novo filtering and reference-based filtering against the UNITE reference dataset v7.1 [22]. Additionally, primer artifacts were filtered out using the PipeCraft built-in module. Full-length ITS2 sequences specific to fungi and oomycete kingdoms were extracted using ITS Extractor v1.0.11 [23] and clustered into operational taxonomic units (OTUs) at 97\% sequence similarity using CD-HIT v4.6 [24]. Single OTUs were removed from further analyses. Prior to clustering, homopolymers $>8 \mathrm{bp}$ were collapsed to $8 \mathrm{bp}$ in all reads to reduce the weight to indels. Taxonomic assignment of OTUs was performed using BLASTn [25] (e-value = 10; word size = 7; reward = 1; penalty = -1 ; gap opening cost $=1$; gap extension cost $=2$ ) against the UNITE + INSD reference dataset $[22,26]$. For unidentified OTUs, manual BLASTn searches were performed in the GenBank database (www.ncbi.nlm.nih.gov). OTUs with an e-value < e50 of the BLASTn search results, BLAST id $\%>70 \%$ and query coverage $>55 \%$ were considered reliable for taxonomy assignments. 


\section{Statistical analysis}

All statistical analyses were performed in R (https://www.r-project.org). QIIME2 and PipeCraft output and sample metadata files were imported into R v4.1.0. Microbial diversity and community composition were analyzed at the ASV or OTU level with the phyloseq [27] and vegan [28] packages in R. The Shannon index was calculated for bacteria/archaea and fungal/protist samples as a measure of the alpha diversity (within sample). Significant differences were tested using the Wilcoxon rank-sum test $(P<0.05)$. To estimate beta diversity (between-sample), nonmetric multidimensional scaling (NMDS) ordination of binary Jaccard dissimilarity matrices was used to identify differences between microbial communities sampled from different country regions and farming systems using PERMANOVA (with 999 permutations) and ANOSIM pairwise comparisons at $P<0.001$. The relative abundance of taxa is depicted using a stacked bar representation with the ASV/OTU mean abundance values expressed as percentages by employing the phyloseq package. Shared taxa of bacteria, archaea, fungi and protists among country regions and farming systems were defined using the VennDiagram R package [29]. To identify taxa, geographical regions and farming systems, differential abundance analysis was carried out using the DESeq2 v1.32.0 package [30]. The FungalTraits database $(P<0.01)[31]$ was used to assign traits (trophic modes) to OTUs assigned to the fungal kingdom (Additional file 4). $P$ values were corrected for multiple comparisons with Dunn's test using the false discovery rate (FDR) correction method [32].

\section{Microbial co-occurrence network analysis}

Co-occurrence networks were constructed and analyzed using the R package NetCoMi v1.0.2 [33] with the functions netConstruct() for network construction, netAnalyze() for network analysis, and netCompare() for network comparison. Associations were estimated based on Pearson's correlation coefficient [34] as an association measure with centered log-ratio (clr) transformation from the SpiecEasi package [35] as a normalization method and the multiplicative replacement (multRepl) method from the zCompositions package [36] to address zeros and missing values in the dataset. Data were filtered to include at least 50 sequences for both the 16S rRNA and ITS2 rDNA gene sequence datasets. Clusters were identified using the default "cluster_fast_greedy" algorithm [37]. Networks were generated using the 50 most abundant taxa to simplify correlation figures. Rhizobium-fungal pathogen cooccurrence networks were created using species-level data from the Rhizobium genus and potential pathogenic groups of fungal genera. Henceforth, potential pathogenic or potentially beneficial microbes are denominated pathogenic or beneficial in the text.

\section{Rhizobium isolation}

Pink nodules, 1-3 mm in diameter, were excised from frozen red clover root samples and rinsed in tap water. The nodules were surface sterilized by submersion in $95 \%$ ethanol for $30 \mathrm{~s}$, followed by $10 \%$ sodium hypochlorite for $90 \mathrm{~s}$ and rinsing with autoclaved water. The following procedures was performed under sterile conditions. Sterilized nodules were suspended in $100 \mu \mathrm{l} \mathrm{H}_{2} \mathrm{O}$ and crushed with a plastic rod until a milky suspension formed. This suspension was cultivated on tryptone yeast and selective yeast mannitol agar (YEMA) plates incubated at $28^{\circ} \mathrm{C}$ in the dark [38]. Colonies were picked after 48 and streaked onto YEMA and Jensen's nitrogen deficient media plates [39] with and without $0.025 \mathrm{~g} / \mathrm{l}$ Congo red dye (Sigma-Aldrich) and incubated at $28^{\circ} \mathrm{C}$ in the dark. Isolates matching the criteria for growth on nitrogen-deficient media and lack of dye absorption were restreaked to obtain pure cultures.

\section{Rhizobium DNA, PCR and sequencing}

Single colonies were grown on YEMA plates, and DNA was isolated [40]. Two fragments of the 16S ribosomal RNA gene were amplified using Phusion High-Fidelity PCR (Thermo Fisher Scientific) with primers (Additional file 2: Table S1). Amplified products were sequenced using Sanger technology (Eurofins Genomics, Ebersberg, Germany). The obtained sequences were analyzed for quality using SnapGene software (from Insightful Science; available at snapgene.com) and assembled with Lasergene MegAlign Pro software (DNASTAR, Inc.). The taxonomy of the assembled sequences was determined using a BLASTN search at NCBI with a minimum of $99 \%$ identity. 


\section{Data availability}

Amplicon sequencing raw datasets have been deposited in the European Nucleotide Archive (ENA) under accession number PRJEB49635. The sequence of a Rhizobium leguminosarum bv. trifolii isolate has been submitted to GenBank at NCBI under accession number MW980046.

\section{Result}

\section{Samples from organic farms contain broad biodiversity}

Two hundred and sixty red clover samples were collected from 89 Swedish field sites. Forty-six samples were derived from 17 organic farms, representing a similar proportion of organic farmland as the official statistics for the entire country (www.sjv.se). Each sample comprised 2 to 4 root systems. DNA isolation was performed mainly as previously described [10] to generate information on root-associated microbiota. In total, 9,944,450 high-quality amplicon sequences from the V3-V4 region of the 16S rRNA gene and 321,703 quality-filtered ITS reads were generated. The 16S rRNA gene sequences were sorted into 53,990 bacterial and 74 archaea amplicon sequence variants (ASVs) after rarefying to 11,184 sequences. The curated ITS dataset was assigned to 2756 fungal and 26 protist operational taxonomic units (OTUs). The dataset was further divided according to geographic origin. The southern region has intense agriculture similar to northern Germany. The northern region reaches the Arctic polar circle with few crops available for agricultural production and where leys are more common. Between those two regions are the central plains.

No significant differences were detected in the alpha diversity (within-sample diversity) of bacteria/archaea communities at the ASV level when comparing samples among the northern, central and southern regions of Sweden (Fig. 1a). The only exception was that the samples from the central region were more diverse than those from the northern and southern regions, which were equally diverse (Wilcoxon rank-sum test, FDR, $P<0.05$ ). Furthermore, diversity was significantly greater in organic than in conventional farms (Additional file 2: Table S2). Fungal/protist diversity at the OTU level did not significantly differ between regions or between farming systems $(P<0.05)$. Nevertheless, samples from the southern region were less diverse than those from the northern and central regions, and samples from organic farms were more diverse than those from conventional farms in our dataset (Fig. 1a and 1b). The bacteria/archaea alpha diversity was approximately 2-fold higher than that of fungi/protists. Analysis of the beta diversity (betweensample diversity) using nonmetric multidimensional scaling (NMDS) ordination based on binary Jaccard distances revealed that bacteria/archaea communities in different regions and farming systems generally clustered together. Only a small fraction of the samples from the northern region and conventional farms were distinct from the corresponding central/southern regions and organic farm samples (Fig. 1c and 1d, Additional file 2: Table S3). Samples made up of fungal/protist communities showed a different pattern, where samples from the northern region were clearly distinct from those from the central and southern regions, whereas a large fraction of the samples from conventional and organic farms formed well-defined groups. Overall, microbial biodiversity was lowest in samples from conventional farms in southern Sweden. This finding may result in less available nutrients for the crops [12].

\section{RAM composition is conditioned by geographic location and farming system}

The root-associated microbiota (RAM) communities were further analyzed at the species level with an abundance value set to $>0.001$ to assess the distribution of unique and shared species between different regions and farming systems. Bacterial species were more abundant, followed by fungal, archaea and protist species (Additional file 2: Fig. S2a and b). Likewise, most unique species representing these four main microbial groups were found in the northern region and in samples from conventional farmland. 
Next, we examined the top 15 most abundant taxa for each microbial group at the phylum, class, genus and species levels (Additional file 2: Fig. S3 to Fig. S6). As expected, Allorhizobium-Neorhizobium-Pararhizobium-Rhizobium (hereafter Rhizobium) was the most prevalent bacterial genus in all samples, followed by Sphingomonas, Mucilaginibacter, Flavobacterium and the unclassified Chloroflexi group KD4-96. Rhizobium was more abundant in samples from the north (56\%) than in those from the central (50\%) and southern regions (38\%). No significant difference was observed in samples from the two farm management systems (Additional file 2: Fig. S6a). The unclassified Rhizobium enriched in the RAM samples (Fig. 2a) had 100\% sequence identity to the Rhizobium leguminosarum bv. trifolii strain, which was independently isolated from nodules on the collected materials. The abundance of Rhizobium leguminosarum bv. trifolii gradually decreased from north (56\%) through central (50\%) to south (43\%), but no major influence of farming systems was observed (Fig. 2b). Genera known to harbor both pathogenic and plant growthpromoting bacterial species, such as the unclassified groups Sphingomonas, Pseudomonas, Flavobacterium, Mucilaginibacter, and Aquabacterium, with the latter being present in water and soil, were abundant in all samples.

Among fungi, Leptodontidium, Cladosporium, Clonostachys and Tetracladium genera known for endophytic, ectomycorrhizal, saprotrophic and mycoparasitic lifestyles according to information from the FungalTraits database (Additional file 3) were frequently observed in all samples but with different distributions between regions and farming systems (Additional file 2: Fig. S6b). At the species level, Leptodontidium and Cadophora species were highly abundant in the northern region and Cladosporium cladosporioides in the central and southern regions (Fig. 2c). In comparison of the farming systems, the incidence of Ceratobasidiaceae sp. and Cadophora sp. were higher in organic samples, and Leptodontidium sp. were more prevalent in samples from conventional farms (Fig. 2d).

We also searched for the presence of pathogenic fungi (Additional file 2: Table S5, mean abundance value $>0.0001$ ). A total of 62 pathogenic fungi from different genera were identified in our dataset. A greater number of pathogenic fungi were found less frequently in samples from the northern region and organic farms. Here, a gradient of increasing abundance from north to south was found for Neonectria sp., Dactylonectria hordeicola, Didymella macrostoma, and Plectosphaerella pauciseptata (Fig. 2c, Additional file 2: Table S5). These species were also more prevalent in samples from conventional farms. For example, Neonectria sp. and Dactylonectria hordeicola had approximately 1.5- and 2-fold higher incidences, respectively (Fig. 2d). Among archaea, the unclassified group of Nitrososphaeraceae sp. was highly abundant together with Woesearchaeales uncultured euryarchaeote, Methanomicrobium uncultured Methanolacinia and Group 1.1c uncultured crenarchaeote in samples from the northern region and organic farms (Additional file 2: Fig. S6a). Cercozoa sp. were the most prevalent protists representing the Rhizaria supergroup (Additional file 2: Fig. S6b). No oomycetes (Stramenopiles) were detected, which was unexpected since Aphanomyces euteiches, Phytophthora pisi and Pythium species, among others, are known as soil-borne pathogens in Sweden [43, 44].

To dissect microbial dynamics, our dataset was implemented in the DESeq2 package. A gradient of species enrichment was found from south to north (Additional file 2: Fig. S7 to S11, Additional file 4). In organic farms, no bacterial species were enriched, whereas only one fungal species (Solicoccozyma terricola) appeared in the analysis (Additional file 2: Fig. S12). We noticed that the bacterial species that were enriched or depleted in our samples mostly had plant growthpromoting or antibacterial properties. For example, Streptomyces nogalater was abundant in all geographic regions, particularly in the northern region, compared with the central and southern regions (Additional file 2: Table S4, Additional file 4). Similar distribution patterns of saprotrophs and endophytes were observed among the fungal communities. A higher number of saprotrophic or endophytic fungi and a lower number of pathogenic species were enriched in the northern region than in the central and southern regions (Additional file 2: Fig. S10, Table S6). In comparisons between the two farm management systems, no fungal pathogens were enriched in the samples from organic farms (Additional file 2: Fig. S12b, Table S6). Taken together, these results highlight two components that greatly impact the population shift between pathogenic and beneficial groups of microbes; geographic location (north) and organic farming are clearly associated with beneficial microbial communities. 
Rhizobium leguminosarum bv. trifolii is a keystone species in the northern region and in organic farms

To gain insights into the co-occurrence patterns of red clover root-associated communities in different regions and farming systems, intrakingdom and interkingdom networks were constructed using the top 50 most abundant species to simplify association networks. Bacterial networks from each region and farming system were more complex and densely connected compared with the fungal networks (Additional file 2: Fig. S13 to S16, Table S7). Three hub taxa were identified in each network. The clustering coefficient was higher in the bacterial networks, in addition to the number of nodes and edge density. The fungal network based on data from conventional farms deviated somewhat from these features with moderately high clustering coefficients (Additional file 2: Table S7). High modularity indicates more structured communities within a network and a greater number of connections within modules than between networks [45]. Thus, fungal networks were found to be more modular than bacterial networks, which were particularly prominent in the northern region. Bacterial networks were dominated by beneficial groups of bacterial species. The interactions among them were almost $50 \%$ positive (co-occurrence). When examining the northern and southern bacterial networks, two hub taxa were formed in the south by Aeromicrobium sp. and Serratia sp., whereas no such hub was detected in the north (Fig. 3a). A major difference between the two regions was the interaction between $R$. leguminosarum bv. trifolii and Aeromicrobium sp. It was strongly positive in the north but negative in the south. In a comparison between northern and central bacterial networks, Actinobacteria sp. was identified as a hub taxon in the north and was strongly associated with Microbacteriaceae and Micrococcaceae species but negatively associated with $R$. leguminosarum bv. trifolii (Additional file 2: Fig. S17a). Furthermore, R. leguminosarum bv. trifolii was also negatively associated with Rahnella 1 sp. in the south but positively in the central network (Additional file 2: Fig. S17b). Strong positive synergy was observed between Rhizobium sp. and Sphingomonas sp. in the south, an interaction that was lacking in the central network.

No hub taxa were detected in the fungal networks when comparing the northern and southern regions (Fig. 3b). In the north, several fungi were not associated with other species, whereas in the south, almost all species were connected, except Gibberella tricincta (former Fusarium tricinctum), a species associated with toxin production on cereals in Europe [46]. In the northern network, G. tricincta was positively associated with Cladosporiaceae sp. (Fig. 3b). When comparing the northern and central networks, Solicoccozyma terricola formed a fungal hub taxon in the north and with Tetracladium furcatum in the central region (Additional file 2: Fig. S18a). A strong positive association was observed between $G$. tricincta and the saprotroph Cladosporium cladosporioides in the north, whereas no such synergy was seen in the central region. Furthermore, the endophytes Leptodontidium sp. and Cadophora sp. were abundant and positively associated with each other in the fungal network from the north (Additional file 2: Fig. S18a). C. cladosporioides was positively associated with the pathogenic group of G. tricincta and D. macrostoma in the south, and this association was not seen in the central network (Additional file 2: Fig. S18b).

In a comparison between conventional and organic networks, no major difference was observed in the percentage of positive association in the bacterial networks. In contrast, the fungal network from conventional farms showed a higher positive association in comparison to the data from organic farms, indicating a higher number of negative interactions (strong competition) among pathogenic or beneficial groups of fungal species. Two bacterial hub taxa, Microbacteriacea sp. and Actinobacteria sp. and two fungal hub taxa, Solicoccozyma terricola and Cladosporium cladosporioides, were detected in the organic networks (Fig. 4a and b).

Comparison of bacterial-fungal interkingdom networks showed that the bacteria formed most of the connections, predominantly with other bacteria. This result is also reflected by the greater abundance of bacterial ASVs compared with fungal OTUs in the samples. Only in the comparison between the northern and southern bacterial-fungal interkingdom networks was one fungal species, Clonostachys rosea, associated with bacterial species affiliated with Rahnella 1, Curtbacterium, Pedobacter and Pseudomonas (Additional file 2: Fig. S19). This fungus has antifungal properties [47], which may influence the surrounding microbes and bacterial clustering. Interactions with $C$. roseae 
fluctuated in our dataset; they were positively associated with Enterobacterales and Aeromicrobium species in the north but negatively associated in the south.

Next, co-occurrence networks were constructed to gain insight into the association complexity between Rhizobium and potential pathogenic fungi in different regions and farming systems (Additional file 2: Table S5). To simplify the association networks, the top 50 most abundant species were included in the analysis. This approach generated different clusters, one with pathogen candidates and the others on Rhizobium species. Unexpectedly, the Rhizobium species were negatively associated with all pathogenic groups of fungi, suggesting competition between them. $R$. leguminosarum bv. trifolii was detected as the hub taxon in the northern and organic networks, whereas no hub taxon was found in the central, south and conventional networks (Fig. 5a and b). In central and southern Sweden, additional legume crops are grown, which most likely influences the Rhizobium community and explains the deviant diversity of Rhizobium species in those regions. Furthermore, a few fungal pathogens showed positive interactions with each other in the red clover root environment independent of the Rhizobium species. These species may play important roles in the persistence of this crop.

\section{Discussion}

Roots are important plant organs, besides providing physical anchoring, and nutrient storage, roots interact with surrounding soil and its inhabitants for water and nutrient uptake. Soil communities are incredibly variable and rich in prokaryotic and eukaryotic organisms. A plethora of factors impact their activities and the dynamic web of interactions between these organisms. Plant roots secrete exudates with a variable composition and microbial inhabitants due to host plant species, age and environmental factors $[49,50]$. Plants have the ability to adapt and to some extent modify the composition of root exudates to suit the needs of individual microbes [51]. Microbial communities are also commonly assembled inside the root tissue as endophytes but become detrimental when colonized by soil-borne pathogens. Bacteria are currently a well-characterized part of the plant microbiome. Representatives of Actinobacteria, Bacteroidetes, and Proteobacteria comprise core phyla of different plant root microbiota across various soil types [52, 53]. These bacteria either colonize the root surface as epiphytes or as endophytes inside the root tissue [54]. A similar phenomenon was observed in our datasets, which were composed of approximately $50 \%$ Proteobacteria, $25 \%$ Actinobacteria, and 12\% Bacteroidetes followed by Verrucomicrobia, Chloroflexi and Patescibacteria, each with a low relative abundance. In a study of soils from field trials located outside Helsinki, Finland, Proteobacteria (25\%), Actinobacteria (20\%), and Bacteroidetes, Acidobacteria and Gemmatimonadetes, each with a relative abundance of approximately $10 \%$, were found with minor differences between samples from organic or fertilizer-treated soils of red clover and timothy [55]. Fewer Proteobacteria and more species from Gemmatimonadetes and Acidobacteria were identified in these field plots than in our data.

Studies of the Lotus japonicus and Mesorhizobium loti model systems have long been essential for our understanding of processes involving nodulation and nitrogen fixation. In L. japonicus - M. loti experiments using field soil from Cologne, Germany, a symbiosis-linked bacterial community was found to have strong plant growth-promoting activities attributed to two orders, Flavobacteriales and Burkholderiales [56]. We identified four other orders with the following main classes: Alphaproteobacteria, Actinobacteria, Bacteroidia and Gammaproteobacteria. In our case, Alphaproteobacteria, a class that includes Rhizobium species, had the highest ASV values in all samples, greatly impacting the taxonomic distribution. Rhizobium have been identified in the rhizosphere of a wide range of plant species and are considered members of the plant core microbiome [57]. The microbial community composition was analyzed under multiple plant-soil conditions. We learned that plant species, including legumes, secrete metabolites that influence the rhizosphere microbiome $[56,58]$. There is also competition between various microbes to become attracted to and establish a community surrounding plant roots and for rhizobia to incite nodule formation followed by the transition to a nitrogen-fixing bacteroid $[59,60,61]$. The genus Rhizobium accommodates 112 species, of which several 
are nonsymbiotic [62]. It is known that nodulating rhizobia harbor genes that are activated by quorum-sensing mechanisms [63]. Metabolites involved in quorum sensing and biofilm formation on root systems are also important community factors [64]. We can only speculate that the Rhizobium species network identified in this work could be a result of a joint population-density action to rapidly colonize red clover roots.

Previous studies have revealed that many members of the genera Streptomyces, Sphingomonas, Pseudomonas, Flavobacterium and Mucilaginibacter play vital roles in producing antibacterial compounds and modulating host performance to stimulate plant growth [65-69]. In the northern region where red clover is grown predominantly and conventional farming is less intense, an abundance of these five bacterial genera associated with plant beneficial traits was found, which may explain the low abundance of pathogenic fungi in these samples.

Organic farming is dependent on animal manure and organic fertilizers in addition to legume crops as major nitrogen sources. This practice is reflected among genera in archaea that dominate in samples from organic farms. Members of Group $1.1 \mathrm{c}$ are ammonia oxidizers of the superphyla TACK, comprising Thaumarchaeota, Crenarchaeota and Korarchaeota, which are known to play key roles in the nitrogen cycle [70, 71]. Other enriched archaea were Methanomicrobium in the Euryarchaeota phylum involved in methane production and anaerobic methane oxidation [72], and Woesearchaeales, a member of another archaeal superphyla (DPANN) present in diverse environments, which are believed to participate in methanogenesis [73]. Members of the above archaea were also more prevalent in the samples from the northern region. Mitigating methane, a very potent greenhouse gas, is presently prioritized in the agricultural sector worldwide. Biochar amendment in soils and paddies to reduce $\mathrm{CH} 4$ emissions is a promising management practice $[74,75]$.

Basic information about soil fungi is limited in comparison to information about bacteria and archaea. Although many OTUs were not assigned a trophic mode in the FungalTraits database, a significant number of OTUs were still annotated to a trophic mode at the genus level. Many fungal species identified in our study belong to genera known to be endophytic, saprotrophic and mycoparasitic on species other than red clover [31]. This was also the case among the pathogenic group where most species were not previously reported on red clover in Sweden. For example, Cylindrocarpon-like species residing in Neonectria, Dactylonectria and Ilyonectria have commonly been associated with cankers, root rots, and decay on hardwood and coniferous trees [76, 77], and Plectosphaerella species are associated with root and collar rots of horticultural crops [78]. The frequently occurring clover rot pathogen $S$. trifoliorum had a very low sequence abundance in our samples. The sexual phase of this fungus occurs aboveground, where apothecia develop from germinating sclerotia shallowly buried in the topsoil. The airborne ascospores initiate infections on stems and leaves, preferably at low temperatures, followed by systemic growth toward the taproot [79]. This life cycle differs from the other pathogens detected in this study, likely explaining the low incidence of this fungus.

Microbial network analysis provides insight beyond microbial diversity per se, allowing us to reveal co-occurrence interactions among our microbial taxa. Keystone or central hub taxa are thought to frequently interact with many other taxa, thereby playing an important role in maintaining organism structure and function that could be crucial for each biological niche studied [80]. A study of the seed microbiome of Brassica napus revealed that a high bacterial diversity expressed by tight and complex bacterial networks affects the colonization ability of newcomers, including either symbionts or pathogens [81]. In the current study, we observed a high bacterial diversity in all samples, and $R$. leguminosarum bv. trifolii was identified as a keystone species in the constructed networks. We also identified independent sets of positively interacting fungi. Fusarium oxysporum is a common root-colonizing fungus with strains that can be neutral, have endophytic properties that induce defense against other soil-borne pathogens or are pathogenic [82]. Its impact on red clover is, however, unclear. In addition, several grass and small-grain cereal pathogens appeared in the network analysis, such as Gibberella tricincta (Fusarium tricinctum) and Fusarium culmorum, which

Page $10 / 22$ 
could indicate that red clover is commonly sown with companion forage species to establish leys during the first year. The potential effects of these fungal pathogens on red clover have not been fully clarified.

\section{Conclusion}

In this study, we aimed to map red clover root-associated microbiota throughout Sweden to improve understanding of the soil diversity and species dynamics involving Rhizobium. Several grass fungal pathogens were identified, among which a majority were previously unknown in Sweden. Fungal species with mycotoxin properties [83] are of major concern. The cooccurring network analysis demonstrated clear enrichment of Rhizobium sp. In the samples and extensive competition with fungal species, not the least of which was exerted by $R$. leguminosarum bv. trifolii. How such interkingdom interactions affect nodulation capacity under field conditions is unknown. A large share of Rhizobium genes is devoted to rhizosphere growth and root colonization, suggesting that successful competition is prioritized to succeed with the next step, symbiosis [84]. The datasets generated in this study, in addition to forming the basis for detailed examination of microbial interactions, may be useful as indicators to estimate future changes in soil microbe communities over time. The annual projected temperature rise is higher in Sweden than the global mean [85]. An estimated increase of up to $0.5^{\circ} \mathrm{C}$ per 10 years leads to rapid changes in climate zones and new constraints for food and feed production, including increasing challenging from pathogens.

\section{Abbreviations}

DPANN: acronym of the names of the first included phyla Diapherotrites, Parvarchaeota, Aenigmarchaeota, Nanohaloarchaeota, and Nanoarchaeota in the DPANN superphylum RAM: root-associated microbiota

\section{Declarations}

\section{Acknowledgments}

We want to thank landowners and extension services throughout Sweden that made the sample collection feasible. We are also grateful to T. Lilja, who isolated Rhizobium leguminosarum bv. trifolii from red clover nodules, and P. Jambagi, who performed 500 BLASTN searches. The authors would like to acknowledge the support of the National Genomics Infrastructure (NGI)/Uppsala Genome Center and UPPMAX for providing assistance in massive parallel sequencing and computational infrastructure.

\section{Author's contributions}

C.D. conceived the study and performed part of the field sampling. S.J. carried out all laboratory work and designed and performed most parts of the bioinformatic analysis. K.P.H. edited the R scripts used for data analysis. L.Ö. identified most field sites, and performed a large part of the sampling. C.D. and S.J. interpreted the data and wrote the manuscript. All authors read and approved the final version of the manuscript.

\section{Funding}

This work was supported by a grant from the Swedish University of Agricultural Sciences, Grogrund Initiative. Sequencing was performed by the SNP\&SEQ Technology Platform in Uppsala. The facility is part of the National Genomics Infrastructure (NGI) Sweden and Science for Life Laboratory. The SNP\&SEQ Platform is also supported by the Swedish Research Council and the Knut and Alice Wallenberg Foundation. Work performed at NGI/Uppsala Genome Center has been funded by RFI/VR and Science for Life Laboratory, Sweden.

\section{Availability of data and materials}


Amplicon sequencing raw datasets have been deposited in the European Nucleotide Archive (ENA) under accession number PRJEB49635. The sequence of a Rhizobium leguminosarum bv. trifolii isolate has been submitted to GenBank at NCBI under accession number MW980046.

\section{Ethics approval and consent to participate}

Not applicable

\section{Consent for publication}

Not applicable

\section{Competing interests}

The authors declare that they have no competing interests.

\section{Author's information}

${ }^{1}$ The Swedish University of Agricultural Sciences, Department of Plant Biology, Uppsala BioCenter, Linnean Center for Plant Biology, P.O. Box 7080, S-75007 Uppsala, Sweden

${ }^{2}$ Lantmännen ek. För. Udda Lundqvists väg 11, Lantmännen Lantbruk, S-26881 Svalöv, Sweden

\section{References}

1. Fróna D, Szenderák J, Harangi-Rákos M. Economic effects of climate change on global agricultural production. Nature Conservation. 2021;44:117-39.

2. FAO. Sustainable food systems-Concept and framework. Rome: Agriculture Organization of the United Nations (FAO). 2018; http://www.fao. org/3/ca2079en/CA2079EN.pdf

3. Vermeulen SJ, Campbell BM, Ingram JSJ. Climate change and food systems. Annu. Rev. Environ. Resour. 2012;37:195-22.

4. Christache S-E, Vuja M, Marin E, Cioacă S-I, Vută M. Organic versus conventional farming - a paradigm for the sustainable development of the European countries. Sustainability. 2018; 10: 4279.

5. Ferreira H, Pinto E, Vasconcelos MW. Legumes as a cornerstone of the transition toward more sustainable agri-food systems and diets in Europe. Front. Sustain. Food Syst. 2021; 5:694121.

6. McKenna P, Cannon N, Conway J, Dooley J Davies WP. Red clover (Trifolium pratense) in conservation agriculture: a compelling case for increased adoption. Int. J. Agaric. Sustain.2018; 16: 342-366.

7. Marshall AH, Collins RP, Vale J, Lowe M. Improved persistence of red clover (Trifolium pratense L.) increases the protein supplied by red clover/grass swards grown over four harvest years. Eur. J. Agron. 2017; 89: 38-45.

8. Official statistics of Sweden. Annual Report 2020. www.sb.se

9. Yli-Mattila T, Kalko G, Hannukkala A, Paavanen-Huhtala S, Hakala K. Prevalence, species composition, genetic variation and pathogenicity of clover rot (Sclerotinia trifoliorum) and Fusarium spp. in red clover in Finland. Eur. J. Plant Pathol. 2010;126:13-27.

10. Hu L, Robert CA, Cadot S, Zhang X, Ye M, Li B, Manzo D, Chervet N, Steinger T, van der Heijden MGA, et al. Root exudate metabolites drive plant-soil feedbacks on growth and defense by shaping the rhizosphere microbiota. Nature Comm. 2018;9:2738. 
11. Berry D, Ben Mahfoudh K, Wagner M, Loy A. Barcoded primers used in multiplex amplicon pyrosequencing bias amplification. Appl. Environ. Microbiol. 2011;77:7846-49.

12. Saghai A, Wittorf $L$, Philippot $L$, Hallin S. Loss in soil microbial diversity constrains microbiome selection and alters the abundance of N-cycling guilds in barley rhizosphere. Appl. Soil Ecol. 2022;169:104224

13. Takahashi S, Tomita J, Nishioka K, Hisada T, Nishijima M. Development of a prokaryotic universal primer for simultaneous analysis of bacteria and archaea using next-generation sequencing. PLoS One. 2014;9:e105592.

14. Halvarsson $P$, Höglund J. Sheep nemabiome diversity and its response to anthelmintic treatment in Swedish sheep herds. Parasites \& Vectors 2021;14:114.

15. Ihrmark K, Bödeker I, Cruz-Martinez K, Friberg H, Kubartova A, Schenck J, Strid Y, Stenlid J, Brandström-Durling M, Clemmensen KE, et al. New primers to amplify the fungal ITS2 region-evaluation by 454-sequencing of artificial and natural communities. FEMS Microbiol. Ecol. 2012;82:666-677.

16. Callahan BJ, McMurdie PJ, Rosen MJ, Han AW, Johnson AJ, Holmes SP. DADA2: high-resolution sample inference from Illumina amplicon data. Nat. Methods. 2016;13:581-583.

17. Quast C, Pruesse E, Yilmaz P, Gerken J, Schweer T, Yarza P, Peplies J, Glöckner FO. The SILVA ribosomal RNA gene database project: improved data processing and web-based tools. Nucleic Acids Res. 2013;41:D590-D96.

18. Price MN, Dehal PS, Arkin AP. FastTree: computing large minimum evolution trees with profiles instead of a distance matrix. Mol. Biol. Evol. 2009;26:1641-50.

19. Katoh K, Standley DM. MAFFT multiple sequence alignment software version 7: improvements in performance and usability. Mol. Biol. Evol. 2013;30:772-80.

20. Anslan S, Bahram M, Hiiesalu I, Tedersoo L. PipeCraft: Flexible open-source toolkit for bioinformatics analysis of custom high-throughput amplicon sequencing data. Mol. Ecol. Res. 2017;17:e234-40.

21. Schloss PD, Westcott SL, Ryabin T, Hall JR, Hartmann M, Hollister EB, Lesniewski RA, Oakley BB, Parks DH, Robinson CJ, Sahl JW. Introducing mothur: open-source, platform-independent, community-supported software for describing and comparing microbial communities. Appl. Envir. Microbiol. 2009;75:7537-41.

22. Nilsson RH, Larsson KH, Taylor AF, Bengtsson-Palme J, Jeppesen TS, Schigel D, Kennedy P, Picard K, Glöckner FO, Tedersoo L, et al. The UNITE database for molecular identification of fungi: handling dark taxa and parallel taxonomic classifications. Nucleic Acids Res. 2019;47:D259-64.

23. Bengtsson-Palme J, Ryberg M, Hartmann M, Branco S, Wang Z, Godhe A, De Wit P, Sánchez-García M, Ebersberger I, de Sousa F, et al. Improved software detection and extraction of ITS1 and ITS 2 from ribosomal ITS sequences of fungi and other eukaryotes for analysis of environmental sequencing data. Methods Ecol. Evol. 2013;4:914-919.

24. Fu L. Niu B, Wu S, Li W. CD-HIT: accelerated for clustering the next-generation sequencing data. Bioinformatics. 2012;28:3150-52.

25. Camacho C, Coulouris G, Avagyan V, Ma N, Papadopoulos J, Bealer K, Madden TL. BLAST+: architecture and applications. Bioinformatics. 2009;10: 421.

26. Cochrane G, Karsch-Mizrachi I, Takagi T and on behalf of the international nucleotide sequence collaboration. The international nucleotide sequence database collaboration. Nucleic Acids Res. 2016;44:D48-50.

27. McMurdie PJ, Holmes S. phyloseq: an R package for reproducible interactive analysis and graphics of microbiome census data. PLoS One. 2013;8:e61217.

28. Oksanen J, Blanchet FG, Kindt R, Legendre P, Minchin PR, O'hara RB, Simpson GL, Solymos P, Stevens MH, Wagner H. Community ecology package. R package version. 2018;2-5.

29. Chen H, Boutros PC. VennDiagram: a package for the generation of highly-customizable Venn and Euler diagrams in R. BMC Bioinfo. 2011;12:1-7. 
30. Love MI, Huber W, Anders S. Moderated estimation of fold change and dispersion for RNA-seq data with DESeq2. Genome Biol. 2014;15:1-21.

31. Põlme S, Abarenkov K, Nilsson RH, Lindahl BD, Clemmensen KE, Kauserud H, Nguyen N, Kjøller R, Bates ST, Baldrian $P$, et al. FungalTraits: a user-friendly traits database of fungi and fungus-like stramenopiles. Fungal Diversity. 2020;105:1-6.

32. Benjamini Y, Hochberg Y. Controlling the false discovery rate: a practical and powerful approach to multiple testing. J. R. Statist. Soc. Ser B-Statist. Methodol. 1995;57:289-300.

33. Peschel S, Müller CL, von Mutius E, Boulesteix AL, Depner M. NetCoMi: network construction and comparison for microbiome data in R. Brief. Bioinform. 2021;22: bbaa290.

34. Pearson K. Determination of the coefficient of correlation. Science. 1909; 30: 23-25.

35. Kurtz ZD, Müller CL, Miraldi ER, Littman DR, Blaser MJ, Bonneau RA. Sparse and compositionally robust inference of microbial ecological networks. PLoS Comp. Biol. 2015;11:e1004226.

36. Palarea-Albaladejo J, Martín-Fernández JA. zCompositionsunder a compositional approach. Chemom. Intell. Lab. Syst. 2015;143:85-96.

37. Clauset A, Newman ME, Moore C. Finding community structure in very large networks. Physical Rev. E. 2004; 70 : 066111.

38. Fred EB, Baldwin IL, McCoy E. Root nodule bacteria and leguminous plants. University of Wisconsin, studies in science. 1932;5:41.

39. Jensen HL. Notes on the Biology of Azotobacter. Proc. Soc. Appl. Bact. 11951;4:89-94.

40. Coton E, Coton M. Multiplex PCR for colony direct detection of Gram-positive histamine- and tyramine-producing bacteria. J. Microbiol. Methods. 2005; 63:296-304.

41. Huws SA, Edwards JE, Kim EJ, Scollan ND. Specificity and sensitivity of eubacterial primers utilized for molecular profiling of bacteria within complex microbial ecosystems. J. Microbiol. Methods. 2007; 70:565-569.

42. Chelius MK, Triplett EW. The diversity of archaea and bacteria in association with the roots of Zea mays L. Microb. Ecol. 2001;41: 252-263.

43. Bodenhausen N, Horton MW, Bergelson J. Bacterial communities associated with the leaves and the roots of Arabidopsis thaliana. PLoS ONE 2013; 8:e56329.

44. Hosseini S, Elfstrand M, Heyman F, Funk Jensen D, Karlsson M. Deciphering common and specific transcriptional immune responses in pea towards the oomycete pathogens Aphanomyces euteiches and Phytophthora pisi. BMC Genom. 2015; 16:627.

45. Lamichhane JR, Durr C, Schwanck AA, Robin M-H, Sarthou J-P, Cellier V, Messean A, Aubertot J-N. Integrated management of damping-off diseases. A review. Agronomy for sustainable development, Springer Verlag/EDP Sciences/INRA. 2017; 37: 25 p. 10.1007/s13593-017-0417-y. hal-01606538

46. Newman MEJ. Modularity and community structure in networks. Proc. Natl. Acad. Sci. USA. 2006;103:8577-8582.

47. Ponts N, Gautier C, Gouzy J, Pinson-Gadis L, Foulongne-Oriol M, Ducos C, Richard-Forget F, Savoie J-M, Zhao C, Barrosa G. Evolution of Fusarium tricinctum and Fusarium avenaceum mitochondrial genomes is driven by mobility of introns and of a new type of palindromic microsatellite repeats. BMC Genom. 2020;21:358

48. Sun Z-B, Li S-D, Ren Q, Xu J-L Lu X, Sun M-H. Biology and applications of Clonostachys rosea. J. Appl. Microbiol. 2020; 129:486-495.

49. Herz K, Dietz S, Gorzolka K, Haider S, Jandt U, Scheel D, Bruelheide H. Linking root exudates to functional plant traits. PLoS One. 2018; 13:e0204128. 
50. Dietz S, Herz K, Doll S, Haider S, Jandt U, Bruelheide H, Scheel D. Semi-polar root exudates in natural grassland communities. Ecol. Evol. 2019; 9: 5526-5541.

51. Vives-Peris, de Ollas C, Gómez-Cadenas, Pérez-Clemente RM. Root exudates: from plant to rhizosphere and beyond. Plant Cell Rep. 2020;.39:3-17.

52. Guttman DS, McHardy AC, Schulze-Lefert P. Microbial genome-enabled insights into plant-microorganism interactions. Nat. Rev. Genet. 2014; 15:797-813.

53. Hacquard S, Garrido-Oter R, González A, Spaepen S, Ackermann G, Lebeis S, McHardy AC, Dangl JL.Knight R, Ley R, Schulze-Lefert P. Microbiota and host nutrition across plant and animal kingdoms. Cell Host Microbe. 2015; 17:603-616.

54. Edwards J, Johnson C, Santos-Medellin C, Lurie E, Podishetty NK, Bhatnagar S, Eisen JA, Sundaresan V. Structure, variation, and assembly of the root-associated microbiomes of rice. Proc. Natl. Acad. Sci. USA. 2015;112:E911E920.

55. Li H, Penttinen P, Mikkonen A, Stoddard F, Lindström K. Response of soil bacterial community diversity and composition to time, fertilization, and plant species in a sub-boreal climate. Front. Microbiol. 2020; 11:1780.

56. Zgadzaj R, Garrido-Oter R, Jensen DB, Koprivova A, Schulze-Lefert P, Radutoiu S. Root nodule symbiosis in Lotus japonicus drives the establishment of distinctive rhizosphere, root, and nodule bacterial com- munities. Proc. Natl. Acad. Sci. USA. 2016; 113:E7996-E8005.

57. Yeoh YK, Paungfoo-Lonhienne C, Dennis PG, Robinson N, Ragan MA, Schmidt S, Hugenholtz P. The core root microbiome of sugarcanes cultivated under varying nitrogen fertilizer application. Environ. Microbiol. 2016; 18: 1338-1351.

58. Tkacz A, Cheema J, Chandra G, Grant A, Poole PS. 2015. Stability and succession of the rhizosphere microbiota depends upon plant type and soil composition. ISME J. 2015; 9:2349-2359.

59. Lardi M, de Campos SB, Purtschert G, Eberl L, Pessi G. Competition experiments for legume infection identify Burkholderia phymatum as a highly competitive $\beta$-rhizobium. Front. Microbiol. 2017; 8:1527.

60. Salas ME, Lozano MJ, Lopez JL, Draghi WO, Serrania J, Torres Tejerizo GA, Albicoro FJ, Nilsson JF, Pistorio M, Del Papa MF, Parisi G, Becker A, Lagares A. Specificity traits consistent with legume-rhizobia coevolution displayed by Ensifer meliloti rhizosphere colonization. Environ. Microbiol. 2017; 19:3423-3438.

61. Boivin S, Ait Lahmidi N, Sherlock D, Bonhomme M, Dijon D, Heulin-Gotty K, Le-Quere A, Pervent M, Tauzin M, Carlsson G, Jensen E, Journet EP, Lopez-Bellido R, Seidenglanz M, Marinkovic J, Colella S, Brunel B, Young P, Lepetit M. Host-specific competitiveness to form nodules in Rhizobium leguminosarum symbiovar viciae. New Phytol. 2020; 226:555-568.

62. Lindström K, Mousavi SA. Effectiveness of nitrogen fixation in rhizobia. Microbial Biotechnol. 2020; 13; $1314-1335$.

63. Sanchez-Contreras M, Bauer WD, Gao M, Robinson JB Downie AJ. Quorum-sensing regulation in rhizobia and its role in symbiotic interactions with legumes Phil. Trans. R. Soc. B. 2007; 362: 1149-1163.

64. Flores-Félix JD, Velázquez E, Martínez-Molina E, González-Andrés F, Squartini A, Rivas R. Connecting the lab and the field: genome analysis of Phyllobacterium and Rhizobium strains and field performance on two vegetable crops. Agronomy. 2021; 11: 1124.

65. Viaene T, Langendries S, Beirinckx S, Maes M, Goormachtig S. Streptomyces as a plant's best friend? FEMS Microbiol. Ecol. 2016;92:fiw119

66. Duran P, Thiergart T, Garrido-Oter R, Agler M, Kemen E, Schulze-Lefert P, Hacquard S. Microbial interkingdom interactions in roots promote Arabidopsis survival. Cell. 2018;175:973.

67. Trivedi P, Leach JE, Tringe SG, Sa T, Singh BK. Plant-microbiome interactions: from community assembly to plant health. Nat. Rev. Microbiol. 2020;11:607-621

Page $15 / 22$ 
68. Liu H, Brettell LE, Qiu Z, Singh BK. Microbiome-mediated stress resistance in plants. Trends Plant Sci. 2020;25:733743.

69. Yin C, Casa Vargas JM, Schlatter DC, Hagerty CH, Hulbert SH, Paulitz TC. Rhizosphere community selection reveals bacteria associated with reduced root disease. Microbiome. 2021; 1:1-8.

70. Guy L, Ettema TJ. The archaeal 'TACK'superphylum and the origin of eukaryotes. Trends Microbiol. 2011; 19:580587.

71. Huang L, Chakrabarti,S, Cooper J. Perez A, John SM, Daroub SH, Martens-Habbena W. Ammonia-oxidizing archaea are integral to nitrogen cycling in a highly fertile agricultural soil. ISME Commun. 2021;1: 19.

72. Evans PN. Boyd JA, Leu AO., Woodcroft BJ, Parks DH, Hugenholtz P, Tyson GW. An evolving view of methane metabolism in the Archaea. Nature Rev. 2019; 17: 219-232.

73. Liu X, Li M, Castelle CJ, Probst AJ, Zhou Z, Pan J, Liu Y, Banfield JF, Gu J-D. Insights into the ecology, evolution, and metabolism of the widespread Woesearchaeotal lineages. Microbiome. 2018; 6:102.

74. Kammann C, Ippolito J, Hagemann N, Borchard N, Cayuela ML. Estaviollo JM, Fuertes-Mendizabal T, Jeffery S, Kern $J$, Novak J, et al. Biochar as a tool to reduce the agricultural greenhouse-gas burden - knowns, unknowns and future research needs. J. Environ. Engineering \& Landscape Manag. 2017; 25:114-139.

75. Nan Q, Xin L, Qin Y, Waqas M, Wu W. Exploring long-term effects of biochar on mitigating methane emissions from paddy soil: a review. Biochar. 2021; 3:125-134.

76. Chaverri C, Salgado Y. Hirooka A, Rossman Y, Samuels GJ. Delimitation of Neonectria and Cylindrocarpon (Nectriae, Hypocreales, Ascomycota) and related genera with Cylindrocarpon-like anamorphs. Stud. Mycol. 2011;68:57-78.

77. Cabral A, Rego C, Nascimento T, Oliveira H, Groenewald JZ, Crous PW. Multi-gene analysis and morphology reveal novel Ilyonectria species associated with black foot disease of grapevines. Fungal Biol. 2012;116:62-80.

78. Carlucci A, Raimondo ML, Santos J, Phillips AJ. Plectosphaerella species associated with root and collar rots of horticultural crops in southern Italy. Persoonia: Mol. Phyl. Evol. Fungi. 2012;28:34.

79. Öhberg H, Ruth P, Bång U. Differential responses of red clover cultivars to Sclerotinia trifoliorum under diverse natural climatic conditions. Plant Pathol. 2008; 57:459-466.

80. Banerjee S, Schlaeppi K, van der Heijden MGA. Keystone taxa as drivers of microbiome structure and functioning. Nat. Rev. Microbiol. 2018;16: 567-576.

81. Rybakova D, Mancinelli R, Wikström M, Birch-Jensen AS, Postma J, Ehlers RU, Goertz S, Berg G. The structure of the Brassica napus seed microbiome is cultivar-dependent and affects the interactions of symbionts and pathogens. Microbiome. 2017;5:104

82. de Lamo FJ, Takken FLW Biocontrol by Fusarium oxysporum using endophyte-mediated resistance. Front. Plant Sci. 2020;11:37.

83. Beccari G, Senatore MT, Tini F, Sulyok M, Covarelli L. Fungal community, Fusarium head blight complex and secondary metabolites associated with malting barley grains harvested in Umbria, Central Italy. Int. J. Food Microbiol. 2018;20:33-42.

84. Wheatley RM, Ford BL, Aroney STN, Knights HE, Ledermann R, East AK, Ramachandran VK, Poole PS Lifestyle adaptations of Rhizobium from rhizosphere to symbiosis. Proc. Natl. Sci. USA. 2020; 117:23823-23834.

85. IPCC, https://www.ipcc.ch

\section{Figures}



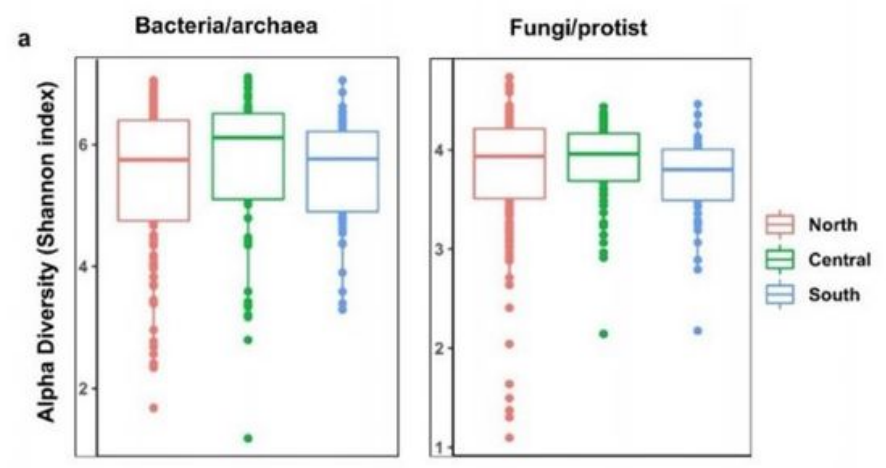

c

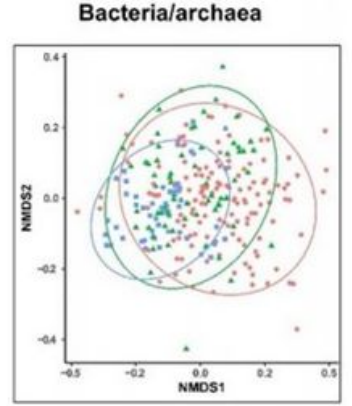

Fungi/protist

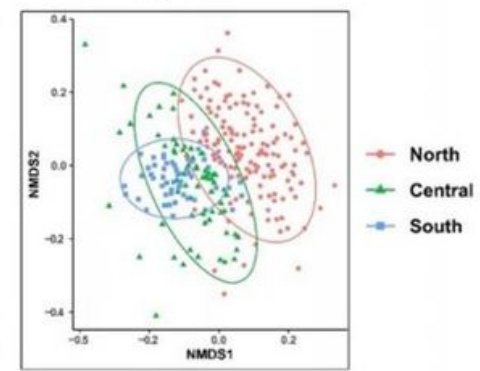

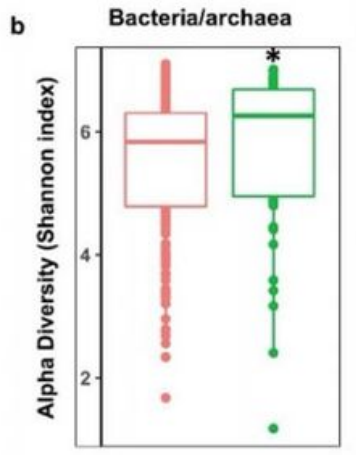

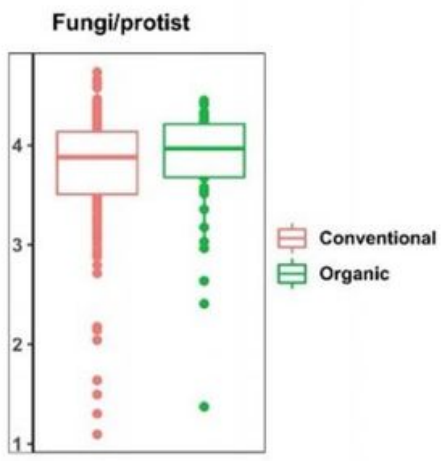

d

Bacteria/archaea

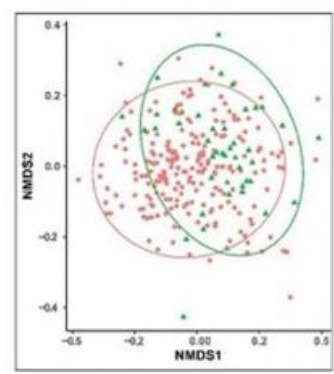

Fungi/protist

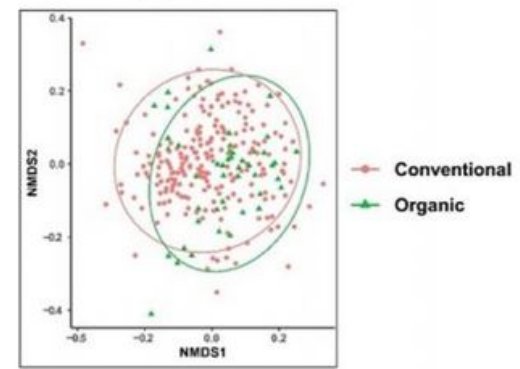

Figure 1

Microbial alpha (within-sample) and beta (between-sample) diversity across different geographic locations and management systems in Sweden. (a) Shannon's alpha diversity of both bacteria/archaea and fungal/protist communities in different regions. (b) Bacteria/archaea and fungal/protist alpha diversity in relation to management systems. The star indicates a significant difference between management systems (Wilcoxon rank-sum test, FDR $P<$ 0.05). Beta diversity of bacteria/archaea and fungal/protist communities in relation to (c) geographic locations and (d) management systems. Beta diversity analysis was based on the nonmetric multidimensional scaling (NMDS) ordination estimated by binary Jaccard distances using PERMANOVA and ANOSIM pairwise comparisons, $P<0.001$. Diversity analyses were conducted at the ASV/OTU level. 
a

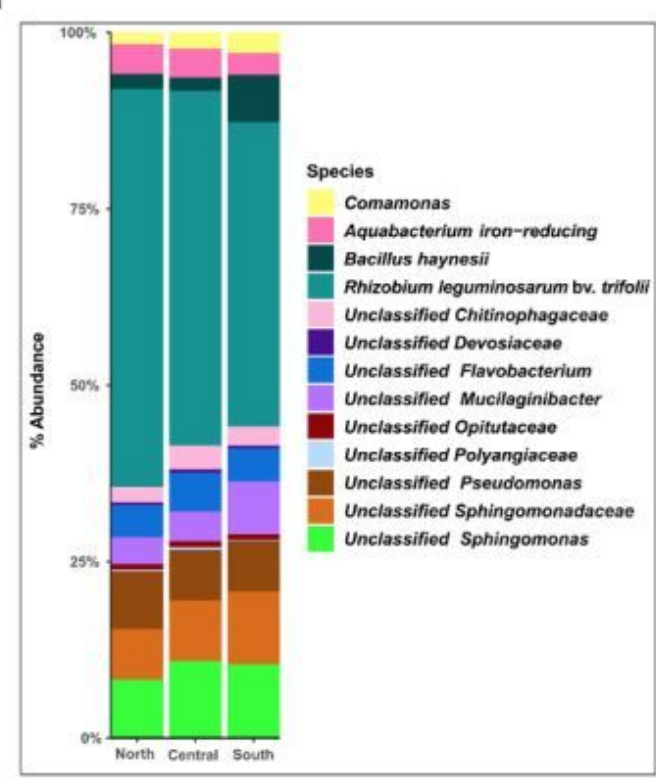

C

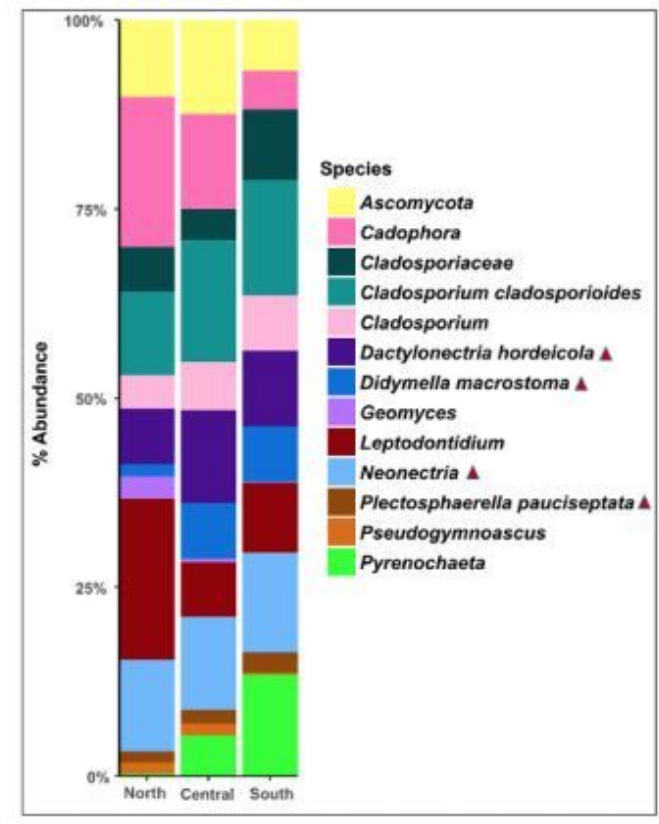

b

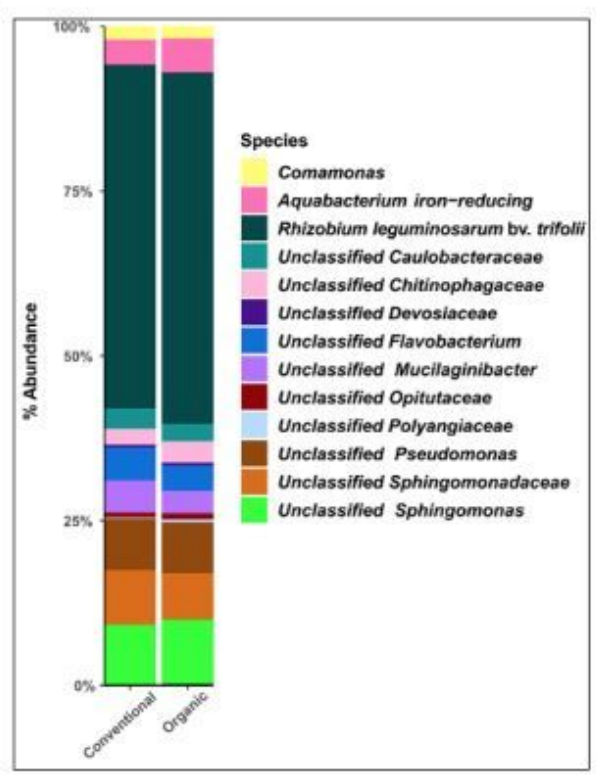

d

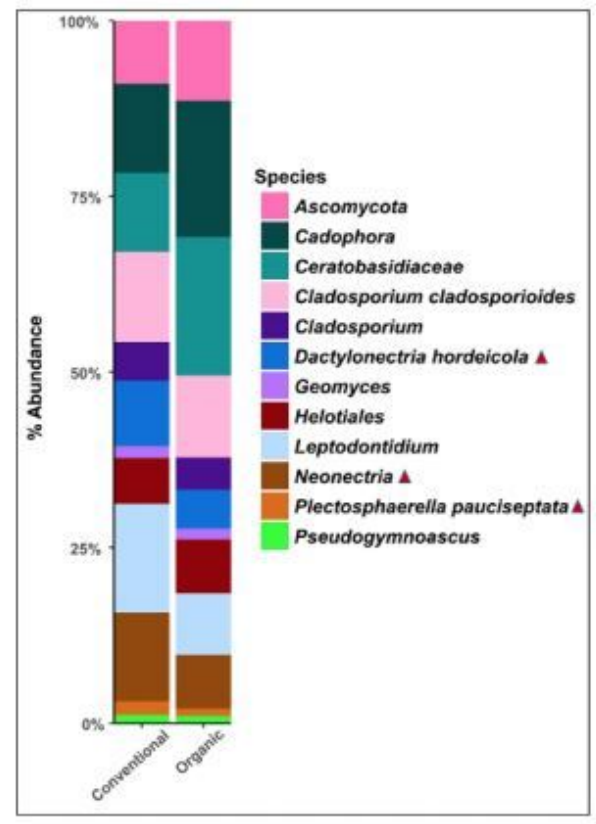

Figure 2

Relative abundances of bacterial and fungal species. Distribution of $(\mathbf{a}, \mathbf{b})$ bacterial and $(\mathbf{c}, \mathbf{d})$ fungal species in samples from different geographic locations and farming systems. "Triangle" denotes the potential pathogenic group of fungi. Low-abundance species $(<1 \%)$ are not illustrated in the bar graphs. 

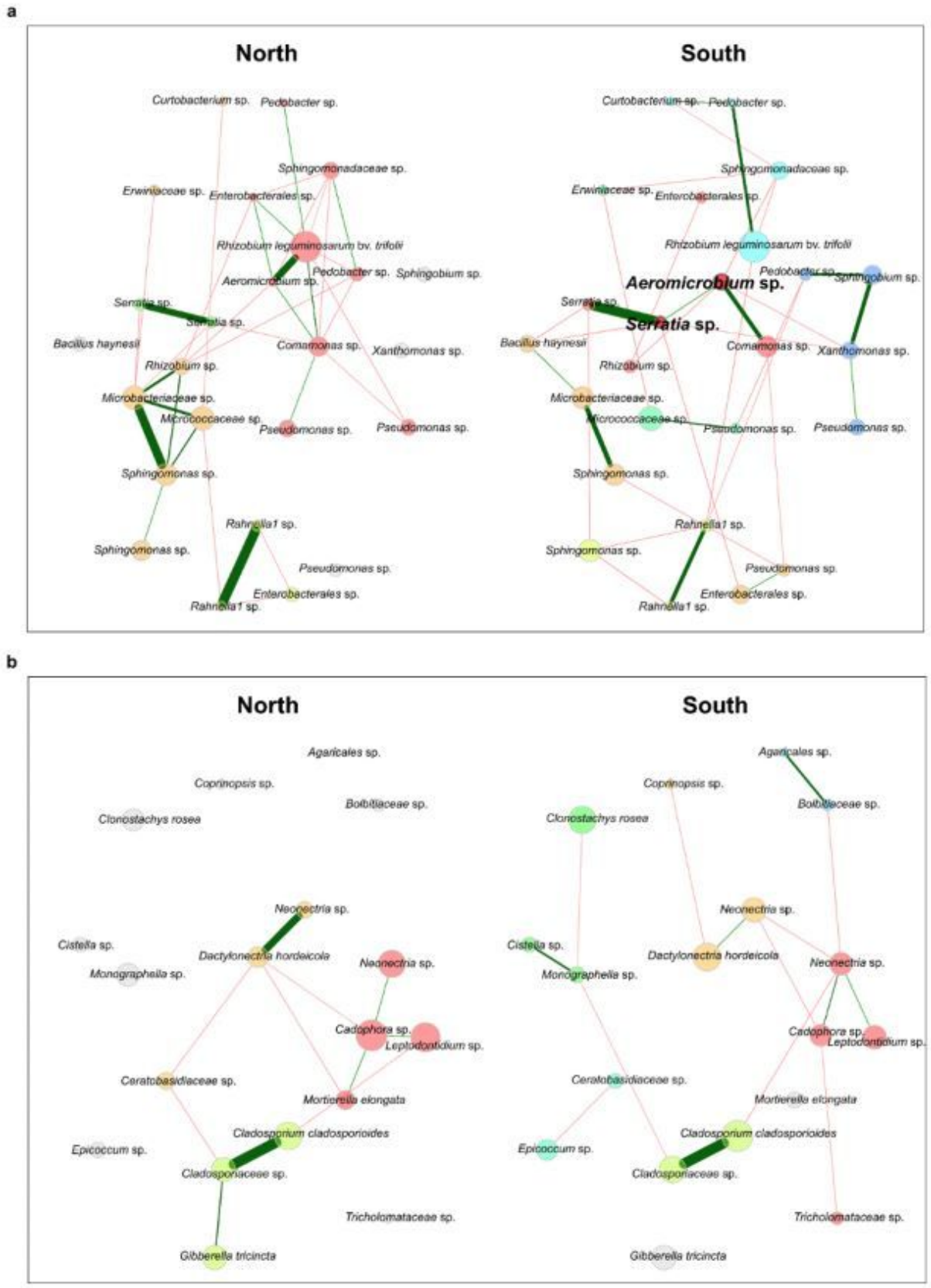

Figure 3

Microbial cooccurrence networks. (a) Bacterial and (b) fungal intrakingdom cooccurrence network based on comparisons between samples from northern and southern regions. Network comparisons were created from specieslevel data based on the Pearson coefficient method [34] using the top 50 most abundant bacterial/fungal species to simplify the association network. Network properties are described in Additional file 2: Table S7. Nodes $=$ species and edges $=$ positive associations (green line) or negative associations (red line). Width/color intensity of lines $=$ strength of positive or negative association. Nodes of the same color are clusters. Size of nodes $=$ relative abundance of that species within a cluster. Hubs are nodes with a centrality value above the empirical $90 \%$ quantile and are identified by bold text. 

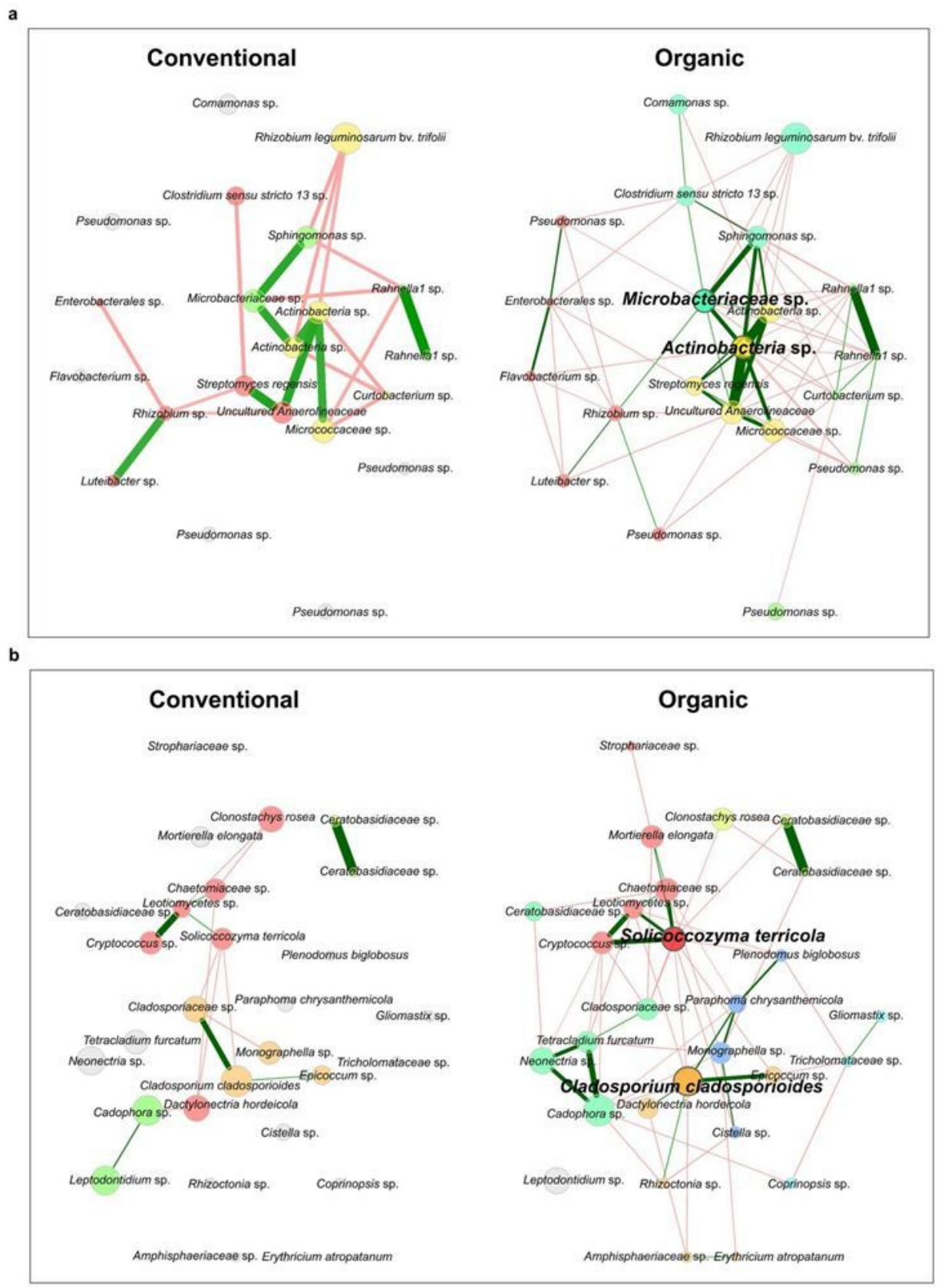

Figure 4

Microbial co-occurrence networks. (a) Bacterial and (b) fungal intrakingdom cooccurrence network comparisons between samples from conventional and organic farming systems. Network comparisons were created from specieslevel data based on the Pearson coefficient method [34] using the top 50 most abundant bacterial/fungal species to simplify the association network. Network properties are described in Additional file 2: Table S7. Nodes $=$ species and edges $=$ positive associations (green line) or negative associations (red line). Width/color intensity of lines $=$ strength of positive or negative association. Nodes of the same color are clusters. Size of nodes $=$ relative abundance of that species within a cluster. Hubs are nodes with a centrality value above the empirical $90 \%$ quantile and are identified by bold text. 

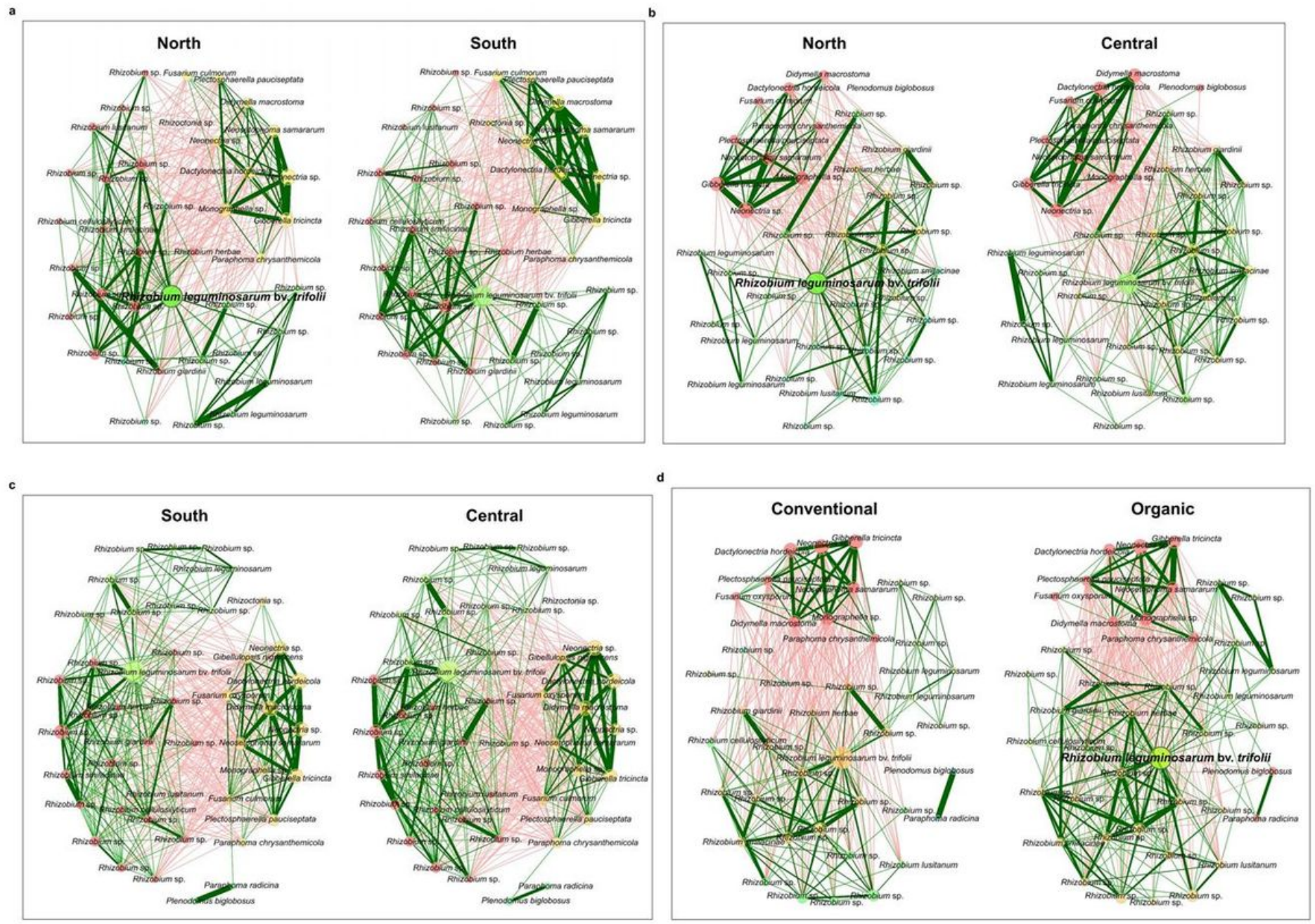

Figure 5

Rhizobium - fungal pathogen cooccurrence networks. Comparisons between samples from (a) northern and southern regions, (b) northern and central regions, (c) southern and central regions and (d) conventional and organic farming systems. Network comparisons were created from Rhizobium species and potential fungal pathogenic group of fungal species level data based on the Pearson coefficient method [34]. Network properties are described in Additional file 2: Table S7. Nodes $=$ species and edges = positive associations (green line) or negative associations (red line). Width/color intensity of lines $=$ strength of positive or negative association. Nodes of the same color are clusters. Size of nodes $=$ relative abundance of specific species within a cluster. Hubs are nodes with a centrality value above the empirical $90 \%$ quantile and are identified by bold text.

\section{Supplementary Files}

This is a list of supplementary files associated with this preprint. Click to download.

- Additionalfile1Samplemetadatainformation.xlsx

- Additionalfile2SupplementaryfiguresandtablesfinalDix.docx 
- Additionalfile3FungallifestyleinformationdeterminedbyqueryinggenusidentitiesagainsttheFungalTraitsdatabase.xlsx

- Additionalfile4Differentialabundanceofrootassociatedbacteriaandfungibetweentheregionsandfarmingsystems.xIsx 